\title{
Reactivity of Nickel metal Precursors towards Amido linked N-heterocyclic Carbenes and their catalytic studies for cross coupling reactions
}

Suryakant Nagar, ${ }^{\mathrm{a}}$ Sucheta Chatterjee, ${ }^{\mathrm{b}}$ Dibakar Goswami, ${ }^{\mathrm{b}}$ c David B. Cordes, ${ }^{\mathrm{d}}$ A. M. Z. Slawin ${ }^{\mathrm{d}}$, Rohit Singh Chauhan, ${ }^{* a}$ Pradnya Prabhu ${ }^{\mathrm{a}}$

aDepartment of Chemistry, K. J. Somaiya College of Science \& Commerce, Mumbai-400077.

Email: rohit.chauhan@somaiya.edu

bio-Organic Division, Bhabha Atomic Research Centre, Anushakti Nagar, Mumbai-400094 and ${ }^{c}$ Homi Bhabha National Institute, Training School Complex, Anushakti Nagar, Mumbai -400 094, India.

Email: dibakarg@,barc.gov.in

${ }^{\mathrm{c}}$ East CHEM School of Chemistry, University of St Andrews, St Andrews, Fife KY16 9ST.

Email: amzs@st-andrews.ac.uk

\begin{abstract}
:
The refluxing of chloroacetamide derivatives with n-butyl imidazole resulted in their corresponding salt of composition 1-( $\underline{n}$-butyl)-3-N-(2-Ar)acetamido-1, 3-imidazolium chloride $(\mathrm{Ar}=$ furylmethyl $(\mathbf{1 a})$; phenylmethyl (1b)). The latter salt on treatment with DBU followed by the addition of chalcogen atom resulted into a compound [(1-( $\underline{n}$-butyl)-3-N-(2Ar)acetamido-1,3-imidazol-3-ylidine $) \mathrm{E}](\mathrm{Ar}=$ furylmethyl; $\mathrm{E}=\mathrm{S}(\mathbf{2 a}) ; \mathrm{Ar}=$ phenylmthyl; $\mathrm{E}=$ Se (2b)). Similarly, the reaction of the imidazolium salts with, excess $\mathrm{K}_{2} \mathrm{CO}_{3}$ and $\mathrm{NiCl}_{2} \cdot 6 \mathrm{H}_{2} \mathrm{O}$ yielded compounds $\quad\left[(1-(\underline{n} \text {-butyl)-3-N-(2-Ar)acetamido-1,3-imidazol-3-ylidine })]_{2} \mathrm{Ni} \quad(\mathrm{Ar}=\right.$ furylmethyl (3a); phenylmethyl (3b)). The latter complexes have also been isolated by using $\mathrm{Ni}(\mathrm{II})$ phosphine precursors $\left[\mathrm{NiCl}_{2}(\mathrm{P}-\mathrm{P})\right]\left(\mathrm{P}-\mathrm{P}=\mathrm{PPh}_{3}\right.$, dppf). The molecular structure of $[(1-(\underline{n}-$ butyl)-3-N-(2-furylmethyl)acetamido-1, 3 -imidazol-3-ylidine $)]_{2} \mathrm{Ni}$ and $[(1-(\underline{n}$-butyl)-3-N-(2-
\end{abstract}


phenylmethyl)acetamido-1, 3-imidazol-3-ylidine) $]_{2} \mathrm{Ni}$ were established by single crystal $\mathrm{X}$-ray diffraction analysis. The catalytic property of complex $\mathbf{3 a}$ and $\mathbf{3 b}$ for cross coupling reactions has been studied.

\section{Introduction:}

The chemistry of N-heterocyclic carbenes (NHC) has become popular due to its applicability in various organic transformations ${ }^{[1-4]}$. These broad prospective interest in NHCs is mainly attributed to their rich structural diversity as well as strong metal carbon bond ${ }^{[5-7]}$. Within the field, internally functionalized amido-linked carbenes have drawn interest due to their catalytic utility for the formation of $\mathrm{C}-\mathrm{X}$ bonds $(\mathrm{X}=\mathrm{C}, \mathrm{N}, \mathrm{P})^{[8-10]}$, biological importance ${ }^{[11,12]}$ and rich coordination chemistry ${ }^{[13,14]}$.

The reaction of [1-R-3\{N-(Arylacetamido)imidazolium\}chloride] $(\mathrm{R}=\mathrm{Me}$, isopropyl, benzyl, menthyl, pinamyl, isboronyl; Aryl = phenyl, benzyl, furyl) with $\mathrm{NiCl}_{2} \cdot 6 \mathrm{H}_{2} \mathrm{O}$ in the presence of $\mathrm{K}_{2} \mathrm{CO}_{3}$ yielded a complex of type $\left\{1-\mathrm{R}-3\right.$ [N-(Arylacetamido)]imidazol-2-ylidene ${ }_{2} \mathrm{Ni}$ where nickel embedded in core of carbene ${ }^{[8,10,15]}$. Even the reaction with 1, 2, 4-triazole derived amido functionalized ligand also resulted in a similar type of compound ${ }^{[16,17]}$. However, the reaction of nickel chloride with symmetrical substituted carbenes afforded $\left[\mathrm{Ni}(\mathrm{NHC})_{2}\right](\mathrm{NHC}=1$, 3-disubstituted imidazol-2-ylidenes) complex which are air stable robust catalyst for cross coupling reactions ${ }^{[18,19]}$. This type of complex has also been reported by Hermann et.al. by the treatment of $\left[\mathrm{NiX}_{2}\left(\mathrm{PPh}_{3}\right)_{2}\right](\mathrm{X}=\mathrm{Cl}, \mathrm{Br})$ with $\mathrm{NHCs}$, displacing the phosphine ligands with the carbene ${ }^{[20,21]}$ and Matsubara et al. have carried out similar reactions to isolate both $\left[\mathrm{NiX}_{2}(\mathrm{NHC})_{2}\right](\mathrm{X}=\mathrm{Cl}, \mathrm{Br})$ and the mixed complex, containing both triphenyl phosphinne and $\mathrm{NHC} ;\left[\mathrm{NiX}\left(\mathrm{PPh}_{3}\right)_{2}(\mathrm{NHC})\right](\mathrm{X}=\mathrm{Cl}, \mathrm{Br} ; \mathrm{NHC}=$ 1,3-bis(2,6-diisopropylphenyl)imidazol-2- 
ylidene) ${ }^{[22]}$. These complexes showed a prominent catalytic reactivity for Grignard cross coupling reactions.

\section{Experimental:}

All manipulations were carried out under a nitrogen atmosphere using standard Schlenk techniques. Solvents used in the reactions were distilled applying standard procedures. The precursor compound N-benzyl and N-furyl 2-chloroacetamide, ${ }^{[23]}$ $\left[\mathrm{NiCl}_{2}(\mathrm{P}-\mathrm{P})\right]\left(\mathrm{P}-\mathrm{P}=\mathrm{PPh}_{3}, \mathrm{dppf}\right),{ }^{[24]}$ and 1-butylimidazole ${ }^{[25]}$ were prepared according to literature methods. Starting materials were procured from Sigma-Aldrich. The ${ }^{1} \mathrm{H}$ and ${ }^{13} \mathrm{C}\left\{{ }^{1} \mathrm{H}\right\}$, ${ }^{77} \mathrm{Se}\left\{{ }^{1} \mathrm{H}\right\}$ NMR spectra were recorded on a Bruker AVANCE--II spectrometer operating at 300, 75.47 and $57.23 \mathrm{MHz}$, respectively. Chemical shifts are relative to internal chloroform peak for ${ }^{1} \mathrm{H},{ }^{13} \mathrm{C}\{1 \mathrm{H}\}$, and external $\mathrm{Ph}_{2} \mathrm{Se}_{2}(\delta 463 \mathrm{ppm})$ in $\mathrm{CDCl}_{3}$ for ${ }^{77} \mathrm{Se}\left\{{ }^{1} \mathrm{H}\right\}$ NMR spectra. Elemental analyses were carried out on a Thermo Fischer Flash EA1112 CHNS analyzer.

The electrochemical experiments were performed using a VERSATAT potentiostat/galvanostat with a three-electrode cell using $\mathrm{Au}$ disk as working electrode, a $\mathrm{Pt}$ gauze as the counter electrode, and a Saturated Calomel electrode as reference electrode. Measurements were made in water with a concentration of $3.0 \mathrm{mmole}^{-1}$ for the nickel complexes. Tetrabutylammonium tetrafluoroborate salt served as electrolyte (concentration 0.12 mmole $\mathrm{L}^{-1}$ ). Potentials were recorded at $\mathrm{mV} \mathrm{s}^{-1}$.

The molecular structure of compound [1-( -butyl)-3-N-(2-furylmethyl)acetamido-1, 3imidazol-3-ylidine) $]_{2} \mathrm{Ni}$ (3a) and [1-( $\underline{n}$-butyl)-3-N-(2-phenylmethyl)acetamido-1,3-imidazol-3ylidine) $]_{2} \mathrm{Ni}$ (3b) were collected at $173 \mathrm{~K}$ on using a Rigaku FR-XUltrahigh Brilliance Microfocus RA generator/confocal optics and XtaLAB P200 diffractometer [Mo- $\mathrm{K}_{\alpha}$ radiation $(\lambda$ $=0.71075 \AA)]$. Intensity data were collected using $\omega$ steps accumulating area detector images 
spanning at least a hemisphere of reciprocal space. Data were collected using Crystal Clear ${ }^{[26]}$ and processed (including correction for Lorentz, polarization and absorption) using CrysAlisPro. The structures were solved by charge-flipping methods (Superflip), ${ }^{[27]}$ and refined by full-matrix least-squares against $F^{2}$ (SHELXL-2018/3). The non-hydrogen atoms were refined anisotropically and hydrogen atoms were refined using a riding model. Molecular structures were drawn using ORTEP. ${ }^{[28]}$ Crystallographic and structural determination data are listed in Table 1. CCDC 1913414-1913415 contains the supplementary crystallographic data for this paper. The data can be obtained free of charge from The Cambridge Crystallographic Data Centre via www.ccdc.cam.ac.uk/structures.

\section{Synthesis of complexes:}

\subsection{Preparation of 1-(n-butyl)-3-N-(2-furylmethyl)acetamido-1,3-imidazolium chloride} (1a)

To a toulene solution of 2-chloro-N-(2-furylmethyl)acetamide (1.5gm, 9.1mmol), n-butyl imidazole $(1.2 \mathrm{ml}, 9.1 \mathrm{mmol})$ was added. The reaction mixture was refluxed for 20 hours at 100 ${ }^{\circ} \mathrm{C}$ which resulted black sticky precipitate. The precipitate was dissolved in minimum volume (5 $\mathrm{mL})$ of dichloromethane which on addition of diethyl ether $(20 \mathrm{~mL})$ yielded a black solid of expected product (yield: 1.2 gm, 53\%; m.p: $62^{\circ} \mathrm{C}$ ) (scheme 1). Anal Calc. for $\mathrm{C}_{14} \mathrm{H}_{20} \mathrm{~N}_{3} \mathrm{O}_{2} \mathrm{Cl}$ : C, 56.46; H, 6.76; N, 14.10\%. Found: C, 56.68; H, 6.86; N, $14.40 \% .{ }^{1} \mathrm{H} \mathrm{NMR}\left(\mathrm{CDCl}_{3}\right) \delta: 9.68(\mathrm{~s}$, $1 \mathrm{H}, \mathrm{NC}(2) H \mathrm{~N}), 9.45$ (br, 1H, NHCO), 7.66 (br, 1H, $\left.\mathrm{C}_{4} H_{3} \mathrm{O}\right), 7.39$ (s, 1H, NC(4)HC), 7.28 (d, J $=4.6 \mathrm{~Hz}, 1 \mathrm{H}, \mathrm{NC}(5) H \mathrm{C}), 6.25\left(\mathrm{~d}, J=7.5 \mathrm{~Hz}, 2 \mathrm{H}, \mathrm{C}_{4} H_{3} \mathrm{O}\right), 5.35\left(\mathrm{~s}, 2 \mathrm{H}, \mathrm{CH}_{2}\right), 4.36(\mathrm{~d}, J=4.8 \mathrm{~Hz}$, $\left.2 \mathrm{H}, \mathrm{CH}_{2} \mathrm{NH}\right), 4.24\left(\mathrm{t}, J=6.9 \mathrm{~Hz}, 2 \mathrm{H},-\mathrm{CH}_{2} \mathrm{CH}_{2} \mathrm{CH}_{2} \mathrm{CH}_{3}\right)$ 1.86-1.81 (m, $\left.2 \mathrm{H},-\mathrm{CH}_{2} \mathrm{CH}_{2} \mathrm{CH}_{2} \mathrm{CH}_{3}\right)$, 1.37-1.30 (m, 2H, $\left.-\mathrm{CH}_{2} \mathrm{CH}_{2} \mathrm{CH}_{2} \mathrm{CH}_{3}\right), 0.92$ (t, $J=7.4 \mathrm{~Hz}, 3 \mathrm{H},-\mathrm{CH}_{2} \mathrm{CH}_{2} \mathrm{CH}_{2} \mathrm{CH}_{3}$ ).

\subsection{Preparation of 1-(n-butyl)-3-N-(2-phenylmethyl)acetamido-1,3-imidazolium chloride} (1b) 
In a method similar to compound $\mathbf{1 a}$, compound $\mathbf{1 b}$ was prepared by using the n-butyl imidazole $(2.0 \mathrm{ml}, 15 \mathrm{mmol})$ and 2-chloro-N-(phenylmethyl)acetamide (2.8 gm, $14.9 \mathrm{mmol})$ giving a white precipitate (yield: 2.4 gm, $66 \%$; m.p: $146^{\circ} \mathrm{C}$ ). Anal Calc. for $\mathrm{C}_{16} \mathrm{H}_{22} \mathrm{~N}_{3} \mathrm{OCl}$ : C, 62.42; H, 7.20; N, 13.65\%. Found: C, 62.96; H, 7.12; N, 13.96\%. ${ }^{1} \mathrm{H}$ NMR $\left(\mathrm{CDCl}_{3}\right) \delta: 9.92(\mathrm{~s}$, $1 \mathrm{H}, \mathrm{NC}(2) H \mathrm{~N}), 9.75(\mathrm{br}, 1 \mathrm{H}, \mathrm{N} H \mathrm{CO}), 7.34-7.16\left(\mathrm{~m}, 7 \mathrm{H}, \mathrm{C}_{6} H_{5}\right), 5.37\left(\mathrm{~s}, 2 \mathrm{H}, \mathrm{CH}_{2}\right), 4.39$ (d, $J=$ $\left.6.0 \mathrm{~Hz}, 2 \mathrm{H}, \mathrm{CH} \mathrm{H}_{2} \mathrm{NH}\right), 4.1\left(\mathrm{t}, J=4.5 \mathrm{~Hz}, 2 \mathrm{H}, \mathrm{CH}_{2} \mathrm{CH}_{2} \mathrm{CH}_{2} \mathrm{CH}_{3}\right), 1.86-1.81(\mathrm{~m}, 2 \mathrm{H}$, $\left.\mathrm{CH}_{2} \mathrm{CH}_{2} \mathrm{CH}_{2} \mathrm{CH}_{3}\right), \quad 1.39-1.32\left(\mathrm{~m}, 2 \mathrm{H},-\mathrm{CH}_{2} \mathrm{CH}_{2} \mathrm{CH}_{2} \mathrm{CH}_{3}\right), \quad 0.95$ (t, $J=7.5 \mathrm{~Hz}, 3 \mathrm{H},-$ $\left.\mathrm{CH}_{2} \mathrm{CH}_{2} \mathrm{CH}_{2} \mathrm{CH}_{3}\right) ;{ }^{13} \mathrm{C}\left\{{ }^{1} \mathrm{H}\right\}$ NMR $\left(\mathrm{CDCl}_{3}\right): 164.8(\underline{\mathrm{C}}=\mathrm{O}), 138.0(\mathrm{~N} \underline{\mathrm{C}}), 137.3(\mathrm{~N}-\underline{\mathrm{C}}(3)-\mathrm{N}), 128.4$ $(\mathrm{Ph}), 127.8(\mathrm{Ph}), 127.1(\mathrm{Ph}), 123.8(\mathrm{Ph}), 120.7(\mathrm{~N}-\underline{\mathrm{C}}(5)-\mathrm{N}), 51.6\left(\underline{\mathrm{CH}_{2}} \mathrm{CH}_{2} \mathrm{CH}_{2} \mathrm{CH}_{3}\right), 49.9(\mathrm{CO}-$

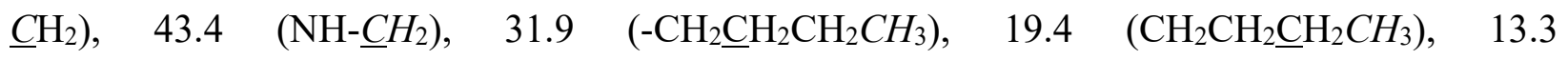
$\left(\mathrm{CH}_{2} \mathrm{CH}_{2} \mathrm{CH}_{2} \mathrm{CH}_{3}\right)$.

\subsection{Preparation of [(1-(n-butyl)-3-N-(2-furylmethyl)acetamido-1,3-imidazol-3-ylidine)S] (2a)}

An acetonitrile solution of 1-( $\underline{n}$-butyl)-3-N-(2-furylmethyl)acetamido-1, 3-imidazolium chloride (261 mg, $1 \mathrm{mmol}$ ), on treatment with 1,8-Diazabicyclo[5.4.0]undec-7-ene (DBU, $0.3 \mathrm{ml}, 2$ $\mathrm{mmol})$ and sulfur powder $(64 \mathrm{mg}, 2 \mathrm{mmol})$ at $50^{\circ} \mathrm{C}$ for 12 hours under inert atmosphere resulted a brown solution. The latter solution was concentrated under reduced pressure which on washing with dichloromethane-acetone mixture afforded 2a (yield: $150 \mathrm{mg}, 51 \%$; m.p.: $88^{\circ} \mathrm{C}$ ) (scheme 1). Anal. Calcd. for $\mathrm{C}_{14} \mathrm{H}_{18} \mathrm{~N}_{3} \mathrm{O}_{2} \mathrm{~S}$ : C, 57.51; H, 6.21; N, 14.37; S, 10.97\%; Found: C, 57.43; H, 6.25; N, 14.11; S, 10.74\%. IR (KBr, cm $\left.{ }^{-1}\right): 3720$ (m), 3173 (w), 3106 (w), 2953 (w), 2926 (w), 2866 (w), 1650 (s), 1547 (s), 1417 (s), 1372 (m), 1336 (m), 1228 (s), 1189 (m), 1143 (m), $1068(\mathrm{~m}), 1012(\mathrm{~m}), 951(\mathrm{w}), 907$ (m), $800(\mathrm{w}), 757(\mathrm{~s}), 706(\mathrm{~m}), 670(\mathrm{~s}), 630(\mathrm{~m}), 602(\mathrm{w})$, 571(w), 528(m). ${ }^{1} \mathrm{H}$ NMR $\left(\mathrm{CDCl}_{3}\right): 7.46$ (br, $\left.1 \mathrm{H}, \mathrm{N} H \mathrm{CO}\right), 7.34\left(\mathrm{~s}, J=2.0 \mathrm{~Hz}, 1 \mathrm{H}, \mathrm{C}_{4} H_{3} \mathrm{O}\right), 6.89$ $(\mathrm{d}, J=4.0 \mathrm{~Hz}, 1 \mathrm{H}, \mathrm{NC}(4) H \mathrm{C}), 6.74(\mathrm{~d}, J=2.0 \mathrm{~Hz}, 1 \mathrm{H}, \mathrm{NC}(5) H \mathrm{C}), \quad 6.30(\mathrm{~d}, J=2.0 \mathrm{~Hz}, 1 \mathrm{H}$, 
$\left.\mathrm{C}_{4} H_{3} \mathrm{O}\right), 6.21\left(\mathrm{dd}, J=3.0 \mathrm{~Hz}\right.$ each, $\left.1 \mathrm{H}, \mathrm{C}_{4} H_{3} \mathrm{O}\right), 4.76\left(\mathrm{~s}, 2 \mathrm{H}, \mathrm{CH}_{2}\right), 4.41(\mathrm{~d}, J=6.0 \mathrm{~Hz}, 2 \mathrm{H}$, $\mathrm{CH}_{2} \mathrm{NH}$ ), 4.05 (t, $\left.J=6.9 \mathrm{~Hz}, 2 \mathrm{H},-\mathrm{CH}_{2} \mathrm{CH}_{2} \mathrm{CH}_{2} \mathrm{CH}_{3}\right)$ 1.69-1.84 (m, $2 \mathrm{H},-\mathrm{CH}_{2} \mathrm{CH}_{2} \mathrm{CH}_{2} \mathrm{CH}_{3}$ ), 1.29-1.44 (m, 2H, - $\left.\mathrm{CH}_{2} \mathrm{CH}_{2} \mathrm{CH}_{2} \mathrm{CH}_{3}\right), 0.93\left(\mathrm{t}, J=6 \mathrm{~Hz}, 3 \mathrm{H},-\mathrm{CH}_{2} \mathrm{CH}_{2} \mathrm{CH}_{2} \mathrm{CH}_{3}\right) ;{ }^{13} \mathrm{C}\left\{{ }^{1} \mathrm{H}\right\} \mathrm{NMR}$ $\left(\mathrm{CDCl}_{3}\right): 167.0(\mathrm{~S}-\mathrm{N} \underline{\mathrm{C}}), 161.7(\underline{\mathrm{C}}=\mathrm{O}), 151.0\left(\underline{C_{4}} \mathrm{H}_{3} \mathrm{O}\right), 141.7\left(\underline{C}_{4} \mathrm{H}_{3} \mathrm{O}\right), 117.5(\mathrm{~N}-\underline{C}(3)-\mathrm{N}), 116.6$ $(\mathrm{N}-\underline{C}(5)-\mathrm{N}), 110.4\left(\underline{C}_{4} H_{3} \mathrm{O}\right), 107.1\left(\underline{C_{4}} H_{3} \mathrm{O}\right), 51.5\left(\underline{\mathrm{CH}}_{2} \mathrm{CH}_{2} \mathrm{CH}_{2} \mathrm{CH}_{3}\right), 48.1\left(\mathrm{CO}-\underline{\mathrm{C}} \mathrm{H}_{2}\right), 36.5(\mathrm{NH}-$ $\left.\mathrm{CH}_{2}\right), 30.9\left(\mathrm{CH}_{2} \mathrm{CH}_{2} \mathrm{CH}_{2} \mathrm{CH}_{3}\right), 19.9\left(\mathrm{CH}_{2} \mathrm{CH}_{2} \mathrm{CH}_{2} \mathrm{CH}_{3}\right), 13.5\left(\mathrm{CH}_{2} \mathrm{CH}_{2} \mathrm{CH}_{2} \mathrm{CH}_{3}\right)$.

2.4 Preparation of [(1-(butyl)-3-N-(2-furylmethyl)acetamido-1,3-imidazol-3-ylidine)Se] (2b)

Prepared similar to above method by using 1-(butyl)-3-N-(furylmethyl)acetamido-1,3imidazolium chloride (261 mg, $1 \mathrm{mmol}$ ), 1,8-diazabicyclo[5.4.0]undec-7-ene (DBU, $0.3 \mathrm{ml}, 2$ mmol) and selenium powder (157.8 mg, $2 \mathrm{mmol}$ ), giving brown powder (yield: $189 \mathrm{mg}, 45 \%$; m.p.: $106^{\circ} \mathrm{C}$ ). Anal. Calcd. for $\mathrm{C}_{14} \mathrm{H}_{18} \mathrm{~N}_{3} \mathrm{O}_{2} \mathrm{Se}$ : C, 49.56; H, 5.35; N, 12.39\%; Found: C, 49.54; H, 5.31; N, 12.19\%. IR (KBr, cm $\left.{ }^{-1}\right): 3271(\mathrm{~m}), 3171(\mathrm{w}), 3142(\mathrm{w}), 3104(\mathrm{w}), 2956(\mathrm{w}), 2926(\mathrm{~m})$, 2864 (w), 1654 (s), 1561 (s), 1544 (s), 1457 (m), 1408 (s), 1373 (m), 1343 (m), 1235 (s), 1139 (m), $1070(\mathrm{~m}), 1012(\mathrm{~m}), 959(\mathrm{w}), 924(\mathrm{w}), 879(\mathrm{w}), 796(\mathrm{w}), 733(\mathrm{~s}), 707(\mathrm{~s}), 666(\mathrm{~s}), 600(\mathrm{w})$, $568(\mathrm{~m}) .{ }^{1} \mathrm{H}$ NMR $\left(\mathrm{CDCl}_{3}\right): 7.45\left(\mathrm{br}, 1 \mathrm{H}, \mathrm{C}_{4} H_{3} \mathrm{O}\right), 7.34(\mathrm{~d}, J=2.0 \mathrm{~Hz}, 1 \mathrm{H}, \mathrm{NC}(4) H \mathrm{~N}), 7.07(\mathrm{~s}, J$ $=2.0 \mathrm{~Hz}, 1 \mathrm{H}, \mathrm{NC}(5) H \mathrm{~N}), 6.89\left(\mathrm{~d}, J=2.2 \mathrm{~Hz}, 1 \mathrm{H}, \mathrm{C}_{4} H_{3} \mathrm{O}\right), 6.28\left(\mathrm{dd}, J=2.8 \mathrm{~Hz}, 1 \mathrm{H}, \mathrm{C}_{4} H_{3} \mathrm{O}\right)$, $6.21\left(\mathrm{~d}, J=3.2 \mathrm{~Hz}, 1 \mathrm{H}, \mathrm{C}_{4} H_{3} \mathrm{O}\right), 4.87\left(\mathrm{~s}, 2 \mathrm{H}, \mathrm{CH}_{2}\right), 4.43\left(\mathrm{~d}, J=6.0 \mathrm{~Hz}, 2 \mathrm{H}, \mathrm{CH}_{2} \mathrm{NH}\right), 4.14(\mathrm{t}, J=$ $\left.7.8 \mathrm{~Hz} 2 \mathrm{H},-\mathrm{CH}_{2} \mathrm{CH}_{2} \mathrm{CH}_{2} \mathrm{CH}_{3}\right), 1.69-1.87\left(\mathrm{~m}, 2 \mathrm{H},-\mathrm{CH}_{2} \mathrm{CH}_{2} \mathrm{CH}_{2} \mathrm{CH}_{3}\right)$. 1.31-1.45 (m, $2 \mathrm{H},-$ $\left.\left.\mathrm{CH}_{2} \mathrm{CH}_{2} \mathrm{CH}_{2} \mathrm{CH}_{3}\right), 0.98\left(\mathrm{t}, J=7.2 \mathrm{~Hz}, 3 \mathrm{H}, \mathrm{CH}_{2} \mathrm{CH}\right)_{3}\right) ;{ }^{13} \mathrm{C}\left\{{ }^{1} \mathrm{H}\right\} \mathrm{NMR}\left(\mathrm{CDCl}_{3}\right): 166.4(\mathrm{Se}-\mathrm{N} \underline{\mathrm{CN}})$, $166.4(\underline{C}=\mathrm{O}), 156.0\left(\underline{C}_{4} H_{3} \mathrm{O}\right), 150.7\left(\underline{C}_{4} H_{3} \mathrm{O}\right), \quad 119.5(\mathrm{~N}-\underline{C}(3)-\mathrm{N}), 119.3(\mathrm{~N}-\underline{C}(5)-\mathrm{N}), 110.3$ $\left(\underline{C}_{4} H_{3} \mathrm{O}\right), 107.4\left(\underline{C_{4}} H_{3} \mathrm{O}\right), 53.1\left(\underline{\mathrm{C}}_{2} \mathrm{CH}_{2} \mathrm{CH}_{2} \mathrm{CH}_{3}\right), 50.0\left(\mathrm{CO}-\underline{\mathrm{C}} \mathrm{H}_{2}\right), 36.5\left(\mathrm{NH}-\underline{\mathrm{C}} \mathrm{H}_{2}\right), 31.11(-$ 


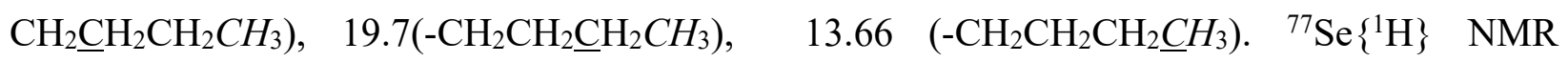
$\left(\mathrm{CDCl}_{3}\right): 6.69 \mathrm{ppm}$.

\subsection{Preparation of [1-(n-butyl)-3-N-(2-furylmethyl)acetamido-1,3-imidazol-3-ylidine) $]_{2} \mathrm{Ni}$}

(3a)

(i) Treatment of an acetonitrile solution of 1-( $\underline{n}$-butyl)-3- $N$-(2-furylmethyl)acetamido-1,3imidazolium chloride $(261 \mathrm{mg}, 1 \mathrm{mmol})$ with $\mathrm{K}_{2} \mathrm{CO}_{3}(1.3 \mathrm{gm}, 10 \mathrm{mmol})$ and $\mathrm{NiCl}_{2} \cdot 6 \mathrm{H}_{2} \mathrm{O}(119$ $\mathrm{mg}, 0.5 \mathrm{mmol}$ ) at $100^{\circ} \mathrm{C}$ for 20 hours resulted in a yellow solution which was concentrated and kept for crystallization under refrigerated conditions to afford a yellow crystalline product (yield: $138 \mathrm{mg}, 27 \%$, m.p.: $175^{\circ} \mathrm{C}$ ). Anal. Calcd. for $\mathrm{C}_{28} \mathrm{H}_{36} \mathrm{~N}_{6} \mathrm{O}_{4} \mathrm{Ni}: \mathrm{C}, 58.05 ; \mathrm{H}, 6.26 ; \mathrm{N}, 14.50 \%$; Found: C, 58.05; H, 6.13; N, 14.21\%. IR (KBr, cm-1) : 3084 (w), 2958 (w), 2873 (w), 1678 (m), $1581(\mathrm{~s}), 1462$ (m), 1427 (m), 1375 (w), 1297 (m), $1241(\mathrm{w}), 1171(\mathrm{~m}), 1144(\mathrm{w}), 1070(\mathrm{w})$, $1001(w), 967$ (w), 930 (w), 884 (w), 803 (w), 738 (s), 679 (m), 630 (w), 599 (m). ${ }^{1} \mathrm{H}$ NMR $\left(\mathrm{CDCl}_{3}\right): 7.26(\mathrm{~s}, 2 \mathrm{H}, \mathrm{NC}(4) H \mathrm{C}), 7.03(\mathrm{~d}, J=2.0 \mathrm{~Hz}, 2 \mathrm{H}, \mathrm{NC}(5) H \mathrm{C}), 6.69$ (d, $J=1.0 \mathrm{~Hz}, 2 \mathrm{H}$, $\left.\mathrm{C}_{4} H_{3} \mathrm{O}\right), 6.27\left(\mathrm{dd}, J=2.0 \mathrm{~Hz}, 2 \mathrm{H}, \mathrm{C}_{4} H_{3} \mathrm{O}\right), 6.03\left(\mathrm{~d}, J=4.0 \mathrm{~Hz}, 2 \mathrm{H}, \mathrm{C}_{4} H_{3} \mathrm{O}\right), 5.25(\mathrm{~d}, J=14 \mathrm{~Hz}$, $\left.2 \mathrm{H}, \mathrm{CH}_{2}\right), 4.86\left(\mathrm{~d}, J=4.0 \mathrm{~Hz}, 2 \mathrm{H}, \mathrm{CH}_{2}\right), 4.40\left(\mathrm{~d}, J=14 \mathrm{~Hz}, 2 \mathrm{H}, \mathrm{CH}_{2}\right), 3.6(\mathrm{~d}, J=14 \mathrm{~Hz}, 2 \mathrm{H}$, $\mathrm{CH}_{2}$ ), 3.20-3.10 (m, 4H, $\left.-\mathrm{CH}_{2} \mathrm{CH}_{2} \mathrm{CH}_{2} \mathrm{CH}_{3}\right)$. 2.79-2.65 (m, $\left.4 \mathrm{H},-\mathrm{CH}_{2} \mathrm{CH}_{2} \mathrm{CH}_{2} \mathrm{CH}_{3}\right), 1.26-1.49(\mathrm{~m}$, 4H, $\left.-\mathrm{CH}_{2} \mathrm{CH}_{2} \mathrm{CH}_{2} \mathrm{CH}_{3}\right), 1.09$ (t, $\left.6.0 \mathrm{~Hz}, 6 \mathrm{H}, \mathrm{CH}_{2} \mathrm{CH}_{3}\right) ;{ }^{13} \mathrm{C}\left\{{ }^{1} \mathrm{H}\right\} \mathrm{NMR}\left(\mathrm{CDCl}_{3}\right): 169.4$ (Ni-N $\underline{\mathrm{C}}$ ), 167.9( $\underline{C}=\mathrm{O}), 156.6\left(\underline{C}_{4} H_{3} \mathrm{O}\right), 140.8\left(\underline{C}_{4} H_{3} \mathrm{O}\right), 122.1 \quad(\mathrm{~N}-\underline{C}(3)-\mathrm{N}), 120.1 \quad(\mathrm{~N}-\underline{C}(5)-\mathrm{N}), 110.2$ $\left(\underline{C}_{4} H_{3} \mathrm{O}\right), 105.9\left(\underline{C}_{4} H_{3} \mathrm{O}\right), \quad 56.6\left(\underline{C H}_{2} \mathrm{CH}_{2} \mathrm{CH}_{2} \mathrm{CH}_{3}\right), 49.3\left(\mathrm{CO}^{-} \mathrm{H}_{2}\right), 41.6\left(\mathrm{NH}-\underline{\mathrm{C}} \mathrm{H}_{2}\right), 32.9$ $\left(\mathrm{CH}_{2} \mathrm{CH}_{2} \mathrm{CH}_{2} \mathrm{CH}_{3}\right), 19.9\left(\mathrm{CH}_{2} \mathrm{CH}_{2} \mathrm{CH}_{2} \mathrm{CH}_{3}\right), 13.4\left(\mathrm{CH}_{2} \mathrm{CH}_{2} \mathrm{CH}_{2} \mathrm{CH}_{3}\right)$.

(ii) To an acetonitrile solution of 1-( $\underline{n}$-butyl)-3-N-(2-furylmethyl)acetamido-1, 3-imidazolium chloride (130mg, $0.5 \mathrm{mmol}), \mathrm{K}_{2} \mathrm{CO}_{3}(1.3 \mathrm{gm}, 10 \mathrm{mmol})$ along with $\left[\mathrm{NiCl}_{2}(\mathrm{dppf})\right]$ (342 mg, 0.5 mmol) were added in the same solvent. The reaction mixture was refluxed at $100^{\circ} \mathrm{C}$ for 14 hours 
and pass through a sintered glass funnel to remove the un-reacted starting materials. The solvent was removed from the filtrate under reduced pressure, and the residue was extracted with dichloromethane-hexane mixture to afford yellow crystals (yield: $105 \mathrm{mg}, 22 \%$; m.p.: $175^{\circ} \mathrm{C}$ ). Anal. Calcd. for $\mathrm{C}_{28} \mathrm{H}_{36} \mathrm{~N}_{6} \mathrm{O}_{4} \mathrm{Ni}$ : C, 58.05; H, 6.26; N, 14.50\%; Found: C, 58.22; H, 6.07; N, 14.36\%. ${ }^{1}\{\mathrm{H}\} \mathrm{NMR}\left(\mathrm{CDCl}_{3}\right):$ 7.26-7.24 (m, $\left.2 \mathrm{H}, \mathrm{NC}(4) H \mathrm{~N}\right), 6.94(\mathrm{~d}, 2.0 \mathrm{~Hz}, 2 \mathrm{H}, \mathrm{NC}(5) H \mathrm{C}), 6.66$ $\left(\mathrm{d}, J=2.0 \mathrm{~Hz}, 2 \mathrm{H}, \mathrm{C}_{4} H_{3} \mathrm{O}\right), 6.27\left(\mathrm{q}, J=4.0 \mathrm{~Hz}, 2 \mathrm{H}, \mathrm{C}_{4} H_{3} \mathrm{O}\right), 6.05\left(\mathrm{~d}, J=4.0 \mathrm{~Hz}, 2 \mathrm{H}, \mathrm{C}_{4} H_{3} \mathrm{O}\right)$, $5.21\left(\mathrm{~d}, J=14 \mathrm{~Hz}, 2 \mathrm{H}, \mathrm{CH}_{2}\right), 4.84\left(\mathrm{~d}, J=16.0 \mathrm{~Hz}, 2 \mathrm{H}, \mathrm{CH}_{2}\right), 4.35\left(\mathrm{~m}, 2 \mathrm{H}, \mathrm{CH}_{2}\right), 3.6(\mathrm{~m}, 4 \mathrm{H}$, $\left.\mathrm{CH}_{2}\right), 3.24-3.14\left(\mathrm{~m}, 4 \mathrm{H},-\mathrm{CH}_{2} \mathrm{CH}_{2} \mathrm{CH}_{2} \mathrm{CH}_{3}\right)$. 2.77-2.66 (m, $\left.4 \mathrm{H},-\mathrm{CH}_{2} \mathrm{CH}_{2} \mathrm{CH}_{2} \mathrm{CH}_{3}\right), 1.57-1.08$ (m, 4H, $\left.-\mathrm{CH}_{2} \mathrm{CH}_{2} \mathrm{CH}_{2} \mathrm{CH}_{3}\right), 0.84\left(\mathrm{t}, J=6.0 \mathrm{~Hz}, 6 \mathrm{H}, \mathrm{CH}_{2} \mathrm{CH}_{3}\right) ;{ }^{13} \mathrm{C}\left\{{ }^{1} \mathrm{H}\right\} \mathrm{NMR}\left(\mathrm{CDCl}_{3}\right): 169.2(\mathrm{Ni}-$ $\mathrm{N} \underline{C} \mathrm{~N}), 168.4(\underline{C}=\mathrm{O}), 156.8\left(\underline{C}_{4} H_{3} \mathrm{O}\right), 140.7\left(\underline{C}_{4} H_{3} \mathrm{O}\right), 121.9(\mathrm{~N}-\underline{C}(3)-\mathrm{N}), 119.9(\mathrm{~N}-\underline{C}(5)-\mathrm{N}), 110.2$ $\left(\underline{C}_{4} H_{3} \mathrm{O}\right), 105.9\left(\underline{C}_{4} H_{3} \mathrm{O}\right), \quad 56.7\left(-\underline{C}_{2} \mathrm{CH}_{2} \mathrm{CH}_{2} \mathrm{CH}_{3}\right), 49.3\left(\mathrm{CO}-\underline{\mathrm{C}} \mathrm{H}_{2}\right), 41.6\left(\mathrm{NH}-\underline{\mathrm{C}} \mathrm{H}_{2}\right), 32.9$ ($\left.\mathrm{CH}_{2} \mathrm{CH}_{2} \mathrm{CH}_{2} \mathrm{CH}_{3}\right), 19.9\left(-\mathrm{CH}_{2} \mathrm{CH}_{2} \underline{\mathrm{CH}_{2}} \mathrm{CH}_{3}\right), 13.4\left(-\mathrm{CH}_{2} \mathrm{CH}_{2} \mathrm{CH}_{2} \underline{\mathrm{CH}} 3\right)$.

(iii) Treatment of an acetonitrile solution of 1-( $\underline{n}$-butyl)-3-N-(phenyl-methyl) acetamido-1, 3imidazolium chloride (130 mg, $0.5 \mathrm{mmol})$ with $\mathrm{K}_{2} \mathrm{CO}_{3}(1.3 \mathrm{gm}, 10 \mathrm{mmol})$, followed by the addition of $\left[\mathrm{NiCl}_{2}\left(\mathrm{PPh}_{3}\right)_{2}\right](327 \mathrm{mg}, 0.5 \mathrm{mmol})$, and heating at $100^{\circ} \mathrm{C}$ for 14 hours resulted I a yellow solution. On passing through a sintered glass filtrate was collected, and solvent was removed under reduced pressure, to obtain yellow powder (yield: $115 \mathrm{mg}, 25 \%$, m.p.: $175^{\circ} \mathrm{C}$ ). Anal. Calcd. for $\mathrm{C}_{28} \mathrm{H}_{36} \mathrm{~N}_{6} \mathrm{O}_{4} \mathrm{Ni}$ : C, 58.05; H, 6.26; N, 14.50\%; Found: C, 58.18; H, 6.27; N, 14.41\%. ${ }^{1} \mathrm{H}$ NMR $\left(\mathrm{CDCl}_{3}\right): 7.34-7.29$ (m, 2H, $\left.\mathrm{NC}(4) H \mathrm{C}\right), 6.97$ (d, $\left.J=2.0 \mathrm{~Hz}, 2 \mathrm{H}, \mathrm{NC}(5) H \mathrm{C}\right)$, $6.67\left(\mathrm{~d}, J=2.0 \mathrm{~Hz}, 2 \mathrm{H}, \mathrm{C}_{4} H_{3} \mathrm{O}\right), 6.30\left(\mathrm{q}, J=4.0 \mathrm{~Hz}, 2 \mathrm{H}, \mathrm{C}_{4} H_{3} \mathrm{O}\right), 6.09(\mathrm{~d}, J=4.0 \mathrm{~Hz}, 2 \mathrm{H}$, $\left.\mathrm{C}_{4} H_{3} \mathrm{O}\right), 5.25\left(\mathrm{~d}, J=14 \mathrm{~Hz}, 2 \mathrm{H}, \mathrm{CH}_{2}\right), 4.88\left(\mathrm{~d}, J=14.0 \mathrm{~Hz}, 2 \mathrm{H}, \mathrm{CH}_{2} \mathrm{NH}\right), 4.39(\mathrm{~d}, J=7.0 \mathrm{~Hz}$, $\left.2 \mathrm{H},-\mathrm{CH}_{2}\right), 3.61\left(\mathrm{~d}, J=4.0 \mathrm{~Hz}, 2 \mathrm{H}, \mathrm{CH}_{2} \mathrm{NH}\right), 3.27-3.12\left(\mathrm{~m}, 4 \mathrm{H},-\mathrm{CH}_{2} \mathrm{CH}_{2} \mathrm{CH}_{2} \mathrm{CH}_{3}\right) . \quad 2.83-2.69$ 
$\left(\mathrm{m}, 4 \mathrm{H},-\mathrm{CH}_{2} \mathrm{CH}_{2} \mathrm{CH}_{2} \mathrm{CH}_{3}\right), 1.41-1.05\left(\mathrm{~m}, 4 \mathrm{H},-\mathrm{CH}_{2} \mathrm{CH}_{2} \mathrm{CH}_{2} \mathrm{CH}_{3}\right) 0.91(\mathrm{t}, J=6.0 \mathrm{~Hz}, 6 \mathrm{H}$, $\left.\mathrm{CH}_{2} \mathrm{CH}_{2} \mathrm{CH}_{2} \mathrm{CH}_{3}\right) ;{ }^{13} \mathrm{C}\left\{{ }^{1} \mathrm{H}\right\} \mathrm{NMR}$ signals are consistent with those noted above.

\subsection{Preparation of [1-(n-butyl)-3-N-(phenyl-methyl)acetamido-1,3-imidazol-3-ylidine) ${ }_{2} N i$} (3b)

(i) Prepared in a similar manner to complex 3a, using method (i), with 1-(n-butyl)-3-N(phenyl-methyl)acetamido-1,3-imidazolium chloride (274 mg, $1 \mathrm{mmol}), \mathrm{K}_{2} \mathrm{CO}_{3}(1.3 \mathrm{gm}, 10$ mmol) and $\mathrm{NiCl}_{2} .6 \mathrm{H}_{2} \mathrm{O}(119 \mathrm{mg}, 0.5 \mathrm{mmol}$ ), giving a yellow powder (yield: $110 \mathrm{mg}, 20 \%$, m.p.: $225^{\circ} \mathrm{C}$ ). Anal. Calcd. for $\mathrm{C}_{32} \mathrm{H}_{40} \mathrm{~N}_{6} \mathrm{O}_{2} \mathrm{Ni}: \mathrm{C}, 64.11 ; \mathrm{H}, 6.72 ; \mathrm{N}, 14.02 \%$; Found: C, 63.86; H, 6.60; N, 14.06\%. IR (KBr, cm-1) : 3117 (w), $2929(\mathrm{w}), 1678(\mathrm{w}), 1578(\mathrm{~s}), 1493$ (w), 1453 (m), $1425(\mathrm{~m}), 1378(\mathrm{~m}), 1338(\mathrm{w}), 1298(\mathrm{~m}), 1237(\mathrm{~m}), 1212(\mathrm{w}), 1175(\mathrm{w}), 1068(\mathrm{w}), 1029(\mathrm{w}), 967$ (w), $913(\mathrm{w}), 806(\mathrm{w}), 737(\mathrm{~m}), 694(\mathrm{~m}), 622(\mathrm{w}) .{ }^{1} \mathrm{H}$ NMR $\left(\mathrm{CDCl}_{3}\right)$ : 7.33-7.08 (m, 10H, $\left.\mathrm{C}_{6} \underline{H}_{5}\right)$, $6.95(\mathrm{~s}, 2 \mathrm{H}, \mathrm{NC}(4) \underline{H C}), 6.66(\mathrm{~d}, J=2.0 \mathrm{~Hz}, 2 \mathrm{H}, \mathrm{NC}(5) \underline{H C}), 5.0\left(\mathrm{~d}, J=14 \mathrm{~Hz}, 2 \mathrm{H}, \underline{\mathrm{H}}_{2}\right), 4.6(\mathrm{~d}$, $\left.J=14 \mathrm{~Hz}, 2 \mathrm{H}, \mathrm{C} \underline{H}_{2}\right), 4.40\left(\mathrm{~d}, J=16 \mathrm{~Hz}, 2 \mathrm{H}, \mathrm{C} \underline{H}_{2}\right), 3.6\left(\mathrm{~d}, J=16 \mathrm{~Hz}, 2 \mathrm{H}, \underline{\mathrm{C}}_{2}\right), 3.07-2.92(\mathrm{~m}$, $\left.4 \mathrm{H}, \quad \underline{\mathrm{C}}_{2} \mathrm{CH}_{2} \mathrm{CH}_{2} \mathrm{CH}_{3}\right), \quad 2.80-2.66 \quad\left(\mathrm{~m}, \quad 4 \mathrm{H}, \quad-\mathrm{CH}_{2} \underline{\mathrm{C}}_{2} \mathrm{CH}_{2} \mathrm{CH}_{3}\right), \quad 1.54-1.28 \quad(\mathrm{~m}, \quad 4 \mathrm{H}$, $\left.\mathrm{CH}_{2} \mathrm{CH}_{2} \underline{\mathrm{CH}}_{2} \mathrm{CH}_{3}\right), 1.20-0.78\left(\mathrm{~m}, 6 \mathrm{H}, \mathrm{CH}_{2} \mathrm{CH}_{2} \mathrm{CH}_{2} \mathrm{C}_{3}\right) ;{ }^{13} \mathrm{C}\left\{{ }^{1} \mathrm{H}\right\}$ NMR $\left(\mathrm{CDCl}_{3}\right): 168.9$ (NiN $\underline{C} \mathrm{~N}), 168.8(\underline{C}=\mathrm{O}), 144.1(\mathrm{~N}-\underline{C}(3)-\mathrm{N}), 128.6(\mathrm{Ph}), 127.9(\mathrm{Ph}), 125.9(\mathrm{Ph}), 121.9(\mathrm{Ph}), 119.5(\mathrm{~N}-$ $\left.\underline{C}(5)-\mathrm{N}), 56.7\left(\underline{\mathrm{CH}}_{2} \mathrm{CH}_{2} \mathrm{CH}_{2} \mathrm{CH}_{3}\right), 49.1(\mathrm{CO}-\underline{\mathrm{CH}})_{2}\right), 49.0\left(\mathrm{NH}-\underline{\mathrm{C}} \mathrm{H}_{2}\right), \quad 32.2\left(-\mathrm{CH}_{2} \underline{\mathrm{CH}}_{2} \mathrm{CH}_{2} \mathrm{CH}_{3}\right)$, $19.9\left(\mathrm{CH}_{2} \mathrm{CH}_{2} \underline{\mathrm{CH}_{2}} \mathrm{CH}_{3}\right), 13.6\left(\mathrm{CH}_{2} \mathrm{CH}_{2} \mathrm{CH}_{2} \underline{\mathrm{CH}_{3}}\right)$.

(ii) Prepared in a similar to compound 3a, using method (ii), with1-(n-butyl)-3-N-(phenylmethyl)acetamido-1,3-imidazolium chloride (137 mg, $0.5 \mathrm{mmol}), \mathrm{K}_{2} \mathrm{CO}_{3}(1.3 \mathrm{gm}, 10 \mathrm{mmol})$ and $\left[\mathrm{NiCl}_{2}(\mathrm{dppf})\right]$ (342 $\left.\mathrm{mg}, 0.5 \mathrm{mmol}\right)$, giving a yellow powder, which was re-crystallized from dichloromethane-hexane (yield: $87 \mathrm{mg}, 18 \%$, m.p.: 225 ${ }^{\circ} \mathrm{C}$ ) (scheme 2). Anal. Calcd. for 
$\mathrm{C}_{32} \mathrm{H}_{40} \mathrm{~N}_{6} \mathrm{O}_{2} \mathrm{Ni}: \mathrm{C}, 64.11 ; \mathrm{H}, 6.72 ; \mathrm{N}, 14.02 \%$; Found: C, 64.32; H, 6.41; N, 14.11\%. ${ }^{1} \mathrm{H}$ and ${ }^{13} \mathrm{C}\left\{{ }^{1} \mathrm{H}\right\}$ NMR signals are consistent with those noted above.

(iii) Prepared in a similar to compound 3a, using method (iii), with 1-(n-butyl)-3-N-(phenylmethyl)acetamido-1,3-imidazolium chloride (137 mg, $0.5 \mathrm{mmol}), \mathrm{K}_{2} \mathrm{CO}_{3}(1.3 \mathrm{gm}, 10 \mathrm{mmol})$ and $\left[\mathrm{NiCl}_{2}\left(\mathrm{PPh}_{3}\right)_{2}\right](327 \mathrm{mg}, 0.5 \mathrm{mmol})$, and heating for 20 hours at $100^{\circ} \mathrm{C}$. This gave a yellow powder which was re-crystallized from dichloromethane-hexane to obtain yellow crystals (yield: 97 mg, 21\%, m.p. $225^{\circ} \mathrm{C}$ ). Anal. Calcd. for $\mathrm{C}_{32} \mathrm{H}_{40} \mathrm{~N}_{6} \mathrm{O}_{2} \mathrm{Ni}$ : C, 64.11; H, 6.72; N, 14.02\%; Found: C, 64.22; H, 6.68; N, 13.98\%. ${ }^{13} \mathrm{C}\left\{{ }^{1} \mathrm{H}\right\}$ NMR signals are consistent with those noted above.

\section{Result and Discussion}

The widening utility of amido functionalized N-heterocyclic carbene as catalysts for base-free Michael addition has led us to prepare our functionalized carbenes ${ }^{[15,23]}$. Refluxing of n-butyl imidazole with 2-chloro-N-(phenylmethyl)acetamide or 2-chloro-N(furylmethyl)acetamide resulted the formation of the 1-( $\underline{n-b u t y l)-3-N-(2-}$ phenylmethyl/furylmethyl)acetamido-1, 3-imidazolium chloride salts in moderate yields (Scheme 1). The formation of the imidazolium salts was verified through ${ }^{1} \mathrm{H}$ NMR by the presence of downfield resonance of protons at 7.28 and $6.25 \mathrm{ppm}$ of 4 and 5 position of the imidazole ring. A blue solution of the free carbene has been generated from these imidazolium salt by the addition of 1,8-diazabicyclo[5.4.0]undec-7-ene (DBU), which on treatment with chalcogens atom afforded a compound of type [(1-( $\underline{n}$-butyl)-3-N-(2-Ar)acetamido-1,3-imidazol3-ylidine)E] $\left(\mathrm{Ar}=\right.$ furyl methyl, $\mathrm{E}=\mathrm{S}$ (2a); Se (2b)) (Scheme 1). The ${ }^{13} \mathrm{C}\left\{{ }^{1} \mathrm{H}\right\}$ NMR of compounds $\mathbf{2 a}$ and $\mathbf{2 b}$ showed a signature resonance of $\mathrm{E}-\mathrm{C}_{\text {carbene }}$ compounds, at $\sim 167 \mathrm{ppm}$. The ${ }^{77} \mathrm{Se}\left\{{ }^{1} \mathrm{H}\right\} \quad \mathrm{NMR}$ of compound [(1-( $\underline{n}$-butyl)-3-N-(2-furylmethyl)acetamido-1,3-imidazol-3- 
ylidine)Se] (2b) displayed a singlet at $6.69 \mathrm{ppm}$ which is comparable to selenium adducts of imidazo[1, 5a]pyridine-3-ylidenes ${ }^{[29]}$. Recently, Ganter et.al. have documented a correlation between the ${ }^{77} \mathrm{Se}$ NMR chemical shift and donor properties of carbene ${ }^{[30]}$. The reports mentioned that de-shielded resonance corresponds to $\pi$-acidic nature of carbene due to the weak interaction of nitrogen lone pair and vacant $\mathrm{p}$ orbital of carbon. However, the selenium adducts 2b resulted a shielded resonance with respect to 1, 3 dialkylimidazole-2-selenone (alkyl = isopropyl, mesityl, fluorophenyl) which resembles poor $\pi$-acceptor ability as well as good donor property of carbene [31]. The IR spectrum of the chalcogen-carbene compounds also demonstrated a characteristic stretching frequency at 1427 and $1570 \mathrm{~cm}^{-1}$ which correspond to the presence of both $\mathrm{E}=\mathrm{C}_{\text {carbene }}(\mathrm{E}=\mathrm{S}, \mathrm{Se})$ and $\mathrm{CO}$ bonds respectively.

The reaction of the substituted carbenes with $\mathrm{NiCl}_{2} \cdot 6 \mathrm{H}_{2} \mathrm{O}$ in the presence of excess $\mathrm{K}_{2} \mathrm{CO}_{3}$ yielded complex of composition [1-(n-butyl)-3-N-(Ar)acetamido-1,3-imidazol-3ylidine) $]_{2} \mathrm{Ni}$ (3) (Ar = Phenylmethyl, (3a); furylmethyl, (3b)) (Scheme 2). It is noteworthy to mention that the similar reaction with $\mathrm{d}^{8}$ system of $\mathrm{Pd}(\mathrm{II})$ precursor results the isolation of complex type $[1-(R)-3-\mathrm{N}$-benzylacetamido-1,3-imidazol-3-ylidine $)]_{2} \mathrm{PdCl}_{2}(\mathrm{R}=$ isopropyl, $\mathrm{CH}_{2} \mathrm{Ph}$ ) ${ }^{[32]}$ where amido linked does not get coordinated with palladium center. The latter discussed outcomes can be rationalized by considering the fact that nickel precursors required quite harsh and stringent condition for the reaction with respect to palladium. Even theoretical calculation of amido linked NHC carbene corresponds 10:1 ratio of $\sigma$ donor $/ \pi$ acceptor property which reflects rich contribution of charge from NHC ligand to metal system ${ }^{[10]}$. A substantial donor nature aroused due to simultaneous chelation of - $\mathrm{NHC}$ carbon to nickel as well as amido nitrogen to nickel center. This chelation through carbon and nitrogen granted an extra stability to the resulting complex. The coordination of nickel is confirmed by the ${ }^{1} \mathrm{H}$ NMR resonances at 
4.96 and $4.63 \mathrm{ppm}$ of imidazole protons. Another prominent characteristic of ${ }^{1} \mathrm{H}$ NMR spectra is the disappearance of proton signal at second position (-NCHN-) of imidazole ring. Additionally, the ${ }^{13} \mathrm{C}\left\{{ }^{1} \mathrm{H}\right\}$ NMR signals correspond to the presence of all expected carbon atoms, similar to those that have been seen previously ${ }^{[8,10]}$. By comparison to the spectra of the free-ligand, a presence of de-shielded resonance at $169 \mathrm{ppm}$ in ${ }^{13} \mathrm{C}\left\{{ }^{1} \mathrm{H}\right\}$ NMR is indicative of the formation of the Ni- $\mathrm{C}_{\text {carbene }}$ bond. The IR spectrum of compounds $\mathbf{3 a}$ and $\mathbf{3 b}$ displayed all vibrations from $3010-550 \mathrm{~cm}^{-1}$. Nevertheless, the intense peaks at 631 and $\sim 690 \mathrm{~cm}^{-1}$ are indicative of the existence of Ni-N and Ni-C carbene bonds respectively ${ }^{[33-36]}$.

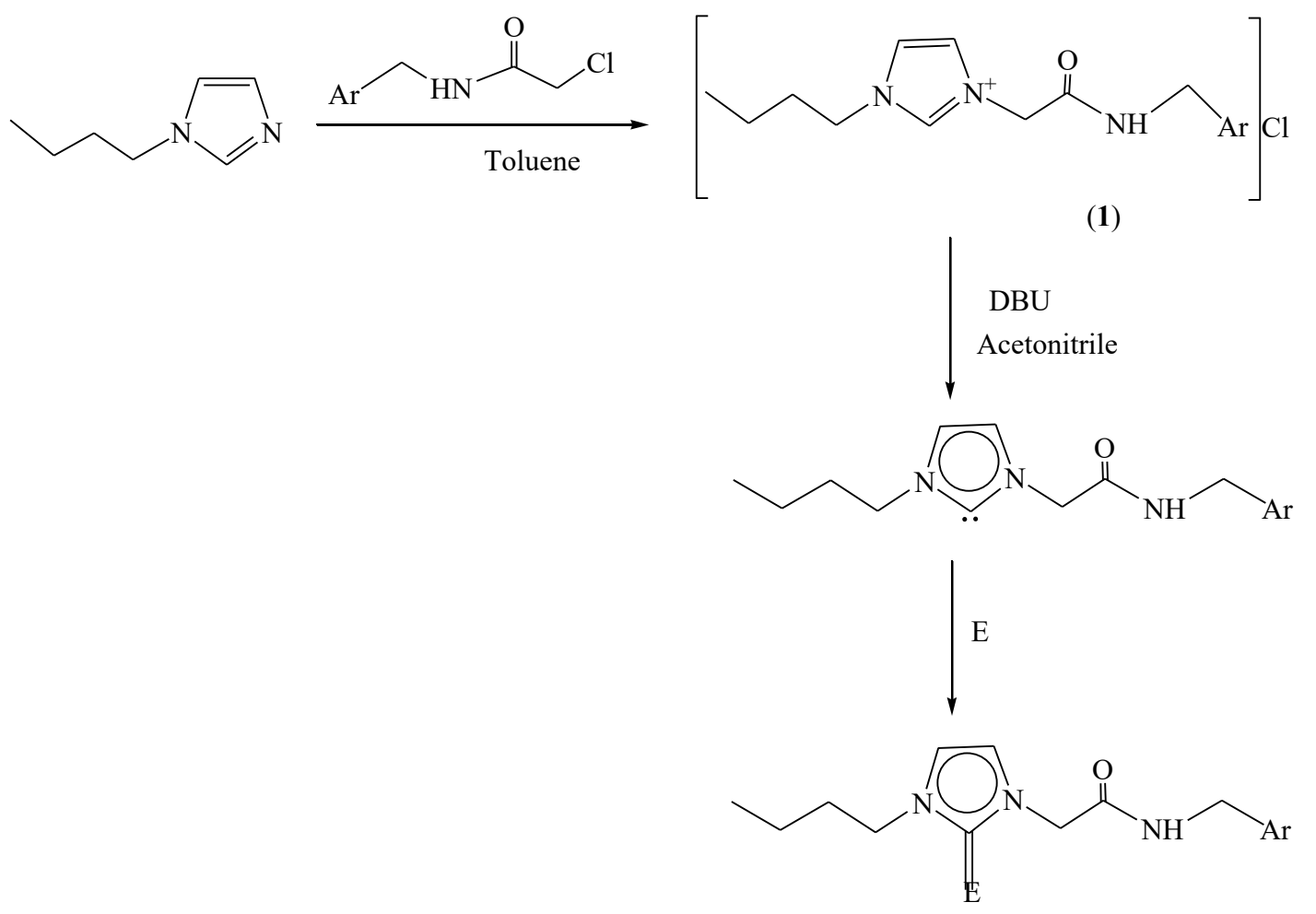

(2)

Scheme 1

\begin{tabular}{l|l|l}
\hline \multicolumn{1}{c|}{$\mathbf{E}$} & \multicolumn{1}{c|}{ Ar } & \\
\hline $\mathrm{S}$ & Furyl & $2 \mathrm{a}$ \\
$\mathrm{Se}$ & Furyl & 2b \\
\hline
\end{tabular}


In a similar manner, the reaction of one equivalent of 1-(n-butyl)-3-N-(2-Ar)acetamido-1, 3-imidazolium chloride $(\mathrm{Ar}=$ Phenylmethyl, furylmethyl $)$ with excess $\mathrm{K}_{2} \mathrm{CO}_{3}$ and $\left[\mathrm{NiCl}_{2}(\mathrm{P}-\mathrm{P})\right]$ $\left(\mathrm{P}-\mathrm{P}=\mathrm{PPh}_{3}\right.$, dppf) also yielded the same compounds $\mathbf{3 a}$ and $\mathbf{3 b}$, respectively. It appears that chelating ring formed by carbon and nitrogen with strong trans effect consequences the substitution of phosphine under the basic condition. The liberation of phosphine due to chelated cyclic ring formed by pyridyl nitrogen and chalcogen center is very well documented whether strong interaction between hard acid -hard base (pyridyl nitrogen) insist the ring formation by removing phosphine in to oxide. ${ }^{[37]}$ An analogous pattern of reactivity has been noted by Grubbs et.al., where various ruthenium carbene complexes having $\mathrm{NHC}$ and $\mathrm{PR}_{3}$ show loss of phosphine ligand under ambient reaction conditions ${ }^{[38,39]}$. In another example, the reaction of $\left[\mathrm{NiCl}_{2}\left(\mathrm{PPh}_{3}\right)\right]$ with 1, 3 di-substituted imidazol-2-ylidenes resulted the substitution of triphenyl phosphine with free carbene. However, partial substitution of $\mathrm{PPh}_{3}$ is also been reported where a reaction of [NiCl $\left.2\left(\mathrm{PPh}_{3}\right)\right]$ with one equivalent of 1,3-bis(2,6-diisopropylphenyl)imidazol-2-ylidene ( $\left.{ }^{i} \mathrm{Pr}\right)$ isolated a compound of type $\left[\mathrm{NiCl}_{2}\left(\mathrm{PPh}_{3}\right)\left({ }^{i} \mathrm{Pr}\right)\right]$ which on keeping for more than one day in solution transformed to $\left[\mathrm{NiCl}_{2}\left(\mathrm{I}^{i} \mathrm{Pr}\right)_{2}\right]^{[22,40]}$. 


\begin{tabular}{l|l}
\hline Furyl & $3 \mathrm{a}$
\end{tabular}

Phenyl 3b

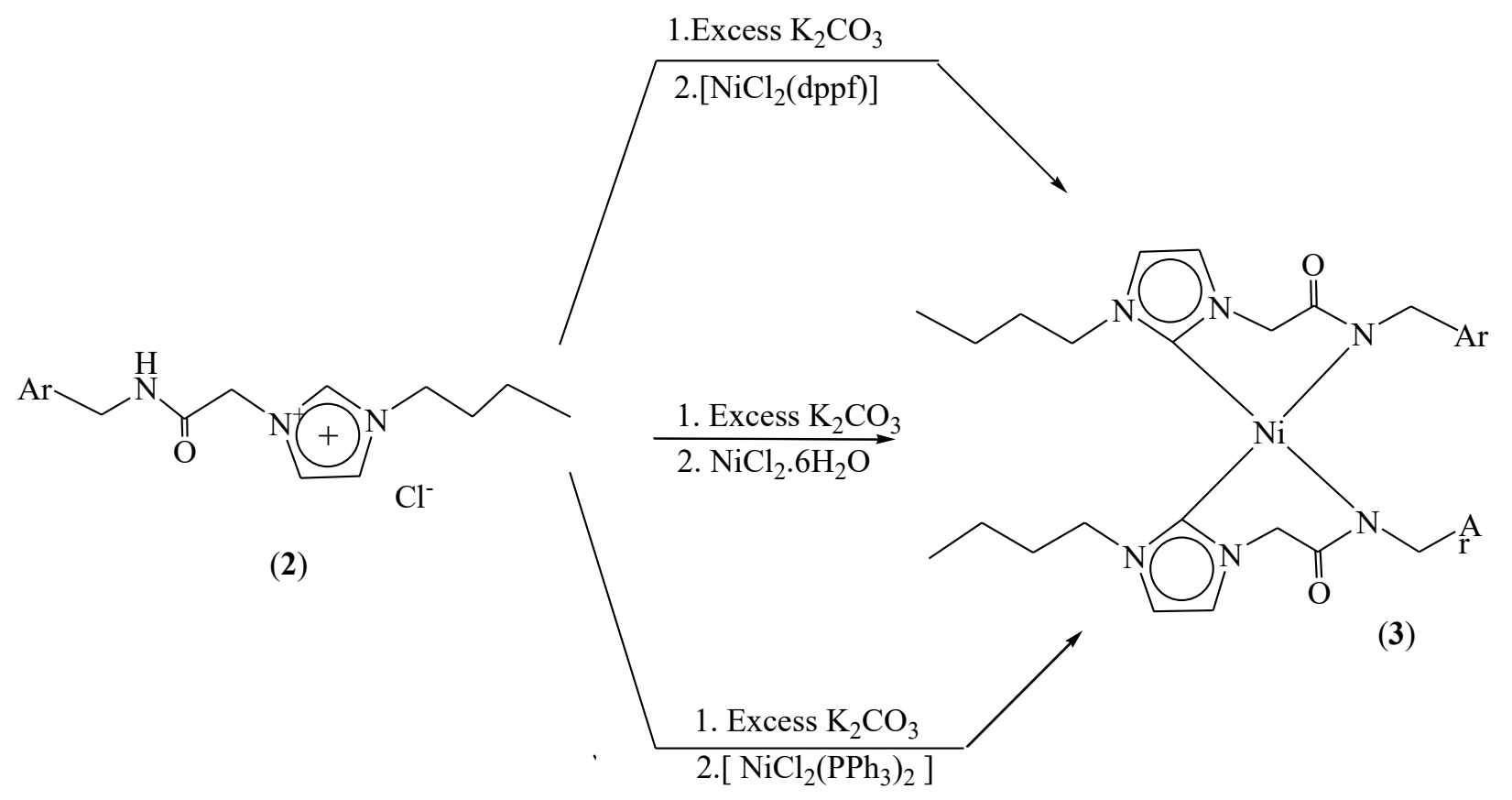

Scheme 2 


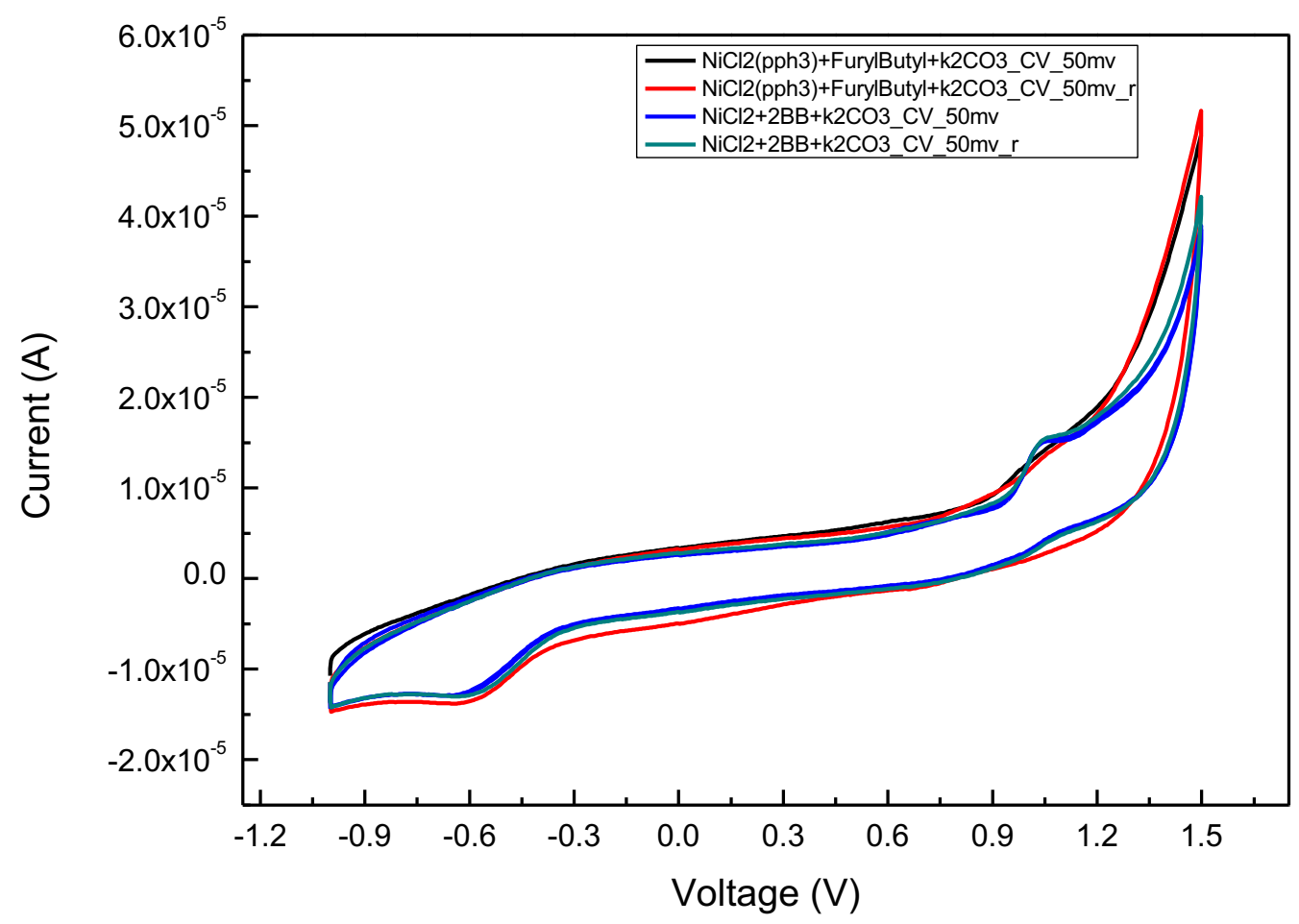

Figure 1: Cyclic voltammogram of complex [1-( $\underline{n}$-butyl)-3-N-(2-furylmethyl)acetamido-1,3imidazol-3-ylidine) $]_{2} \mathrm{Ni}$ (3a) and [1-( $\underline{n}$-butyl)-3-N-(2-phenylmethyl)acetamido-1,3-imidazol-3ylidine) $]_{2} \mathrm{Ni}(\mathbf{3 b})$

The electrochemical behaviour of the $\mathrm{Ni}$ (II) complexes was investigated in $\mathrm{H}_{2} \mathrm{O}$ by cyclic voltammetry $(\mathrm{CV})$. The nature of cyclic voltammogram is reversible in nature and reduction potential at approx. $-0.6 \mathrm{~V}$ is related to the redox $\mathrm{Ni}$ centre in the catalyst, and it may be inferred that the catalytic activities of the catalysts are due to the presence of $\mathrm{Ni}(\mathrm{II})$. With consecutive scans, it was found that the redox centre was stable, and this accounts for the sustained catalytic activity of the complexes.

The solid state structures of [1-( $\underline{n}$-butyl)-3-N-(2-furylmethyl)acetamido-1,3-imidazol-3ylidine) $]_{2} \mathrm{Ni}$ (3a) (figure 2) and [1-( $\underline{n}$-butyl)-3-N-(2-phenylmethyl)acetamido-1,3-imidazol-3- 
ylidine) $]_{2} \mathrm{Ni}\left(\mathbf{3 b}\right.$ ) (figure 3) show similar square planar geometries, with a $\mathrm{C}_{2} \mathrm{~N}_{2}$ coordination environment, the imidaozle rings placed cis to each other. The bond lengths of $\mathrm{Ni}-\mathrm{C}_{\text {carbene }}$ in complex 3a and $\mathbf{3 b}$ is $[1.8658(13) \AA$ and $1.861(2) / 1.859(2) \AA]$, are comparable to the related compound $\quad\left\{1-\left[\left(2\right.\right.\right.$-furanylmethyl)aminocarbonylmethyl]-3-methylimidazol-2-ylidene ${ }_{2} \mathrm{Ni}$ $[1.865(4) \AA, 1.867(5) \AA]$ and longer than [1-(benzyl)-3-N-(benzylacetamido)imidazol-2ylidene $]_{2} \mathrm{Ni}\left[1.841(4) \AA\right.$ and $1.858(4) \AA{ }^{[8,10]}$. The Ni-N bond lengths $[1.9297(11) \AA$ and $1.9306(19) / 1.9367(19) \AA ̊$ are in good agreement with previously reported values from related complexes ${ }^{[7,10,15,16]}$. The bond angles around the nickel in compound $\mathbf{3 a}$ and $\mathbf{3 b}$ varies from $86.58(5)^{\circ}-93.12(12)^{\circ}$, comparable to the analogous compound [1-(benzyl)-3-N(benzylacetamido)imidazol-2-ylidene $]_{2} \mathrm{PdCl}_{2}\left[90.4(8)^{\circ}\right.$ and $\left.90.6(2)^{\circ}\right]{ }^{[32]}$ and $[1-($ benzyl)-3-N(benzylacetamido)imidazol-2-ylidene $]_{2} \mathrm{Ni}^{[8]}$.

\section{Catalytic activities of $3 a$ and $3 b$ in Suzuki cross coupling reactions}

To a stirred solution of the substrate $(1 \mathrm{mmol}$, Table 2$)$ in dioxane $(4 \mathrm{~mL})$ was added phenylboronic acid (0.30 g, $2.5 \mathrm{mmol}), \mathrm{K}_{2} \mathrm{CO}_{3}(0.41 \mathrm{~g}, 3 \mathrm{mmol}), \mathrm{PPh}_{3}(10 \mathrm{~mol} \%)$ and $\mathbf{3 a} / \mathbf{3 b}(2$ mol\%), and was refluxed for $48 \mathrm{~h}$. The reaction mixture was concentrated in vacuo, and the residue was subjected to column chromatography (silica gel, 0-5\% EtOAc/hexane) to obtain respective products. The yields of the products are depicted in Table 2 .

i) Biphenyl (5a): white solid (m.p. $70{ }^{\circ} \mathrm{C}$; lit. $\left.70.5-72{ }^{\circ} \mathrm{C}\right){ }^{[41]}$; ${ }^{1} \mathrm{H}$ NMR $(200 \mathrm{MHz}$, $\left.\mathrm{CDCl}_{3}\right) \delta$ 7.29-7.50 (m, 6H), 7.55-7.66 (m, 4H).

ii) 4-Methoxy-1,1'-biphenyl (5b). white solid (m.p. $91{ }^{\circ} \mathrm{C}$; lit. $91.1-92.3{ }^{\circ} \mathrm{C}$ ) ${ }^{[41]}$; $1 \mathrm{H}$ NMR (200 MHz, $\left.\mathrm{CDCl}_{3}\right) \delta 3.97(\mathrm{~d}, \mathrm{~J}=4.86 \mathrm{~Hz}, 3 \mathrm{H}), 7.03-7.18(\mathrm{~m}, 1 \mathrm{H}), 7.31-7.40$ (m, 1H), 7.45-7.65 (m, 4H), 7.67-7.82 (m, 3H) 
iii) 4-Methyl-1,1'-biphenyl (5c). white solid (m.p. $45^{\circ} \mathrm{C}$; lit. $\left.45-50{ }^{\circ} \mathrm{C}\right){ }^{[41]} ;{ }^{1} \mathrm{H}$ NMR $(200$ $\left.\mathrm{MHz}, \mathrm{CDCl}_{3}\right): \delta 2.41$ (s, 3H), 7.33-7.42 (m, 4H), 7.43-7.60 (m, 5H).<smiles>[R]c1ccc(Br)cc1</smiles><smiles>OBc1ccccc1</smiles>
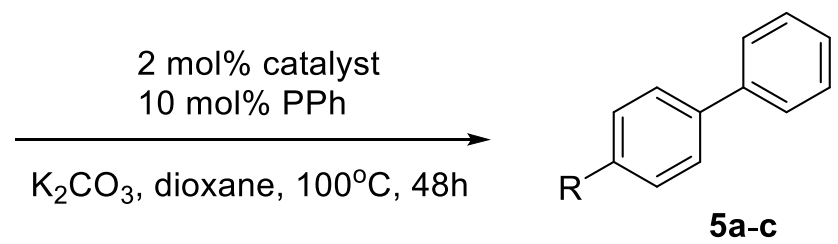

\section{Scheme 3 .}

Table 2. Suzuki coupling of aryl bromides with phenylboronic acid. ${ }^{\mathrm{a}}$

\begin{tabular}{ccccc}
\hline Substrate & $\mathrm{R}$ & Product & Catalyst & Yield $^{\mathrm{b}}$ \\
\hline $\mathbf{4 a}$ & $\mathrm{H}$ & $\mathbf{5 a}$ & $\mathbf{3 a}$ & 60 \\
& & & $\mathbf{3 b}$ & 55 \\
\hline $\mathbf{4 b}$ & $-\mathrm{OCH}_{3}$ & $\mathbf{5 b}$ & $\mathbf{3 a}$ & 65 \\
& & & $\mathbf{3 b}$ & 68 \\
\hline $\mathbf{4 c}$ & $-\mathrm{CH}_{3}$ & $\mathbf{5 c}$ & $\mathbf{3 a}$ & 54 \\
& & & $\mathbf{3 b}$ & 58
\end{tabular}

aReaction conditions: $1 \mathrm{mmol}$ aryl bromide, $2.5 \mathrm{mmol}$ phenylboronic acid, $10 \mathrm{~mol} \% \mathrm{PPh}_{3}, 3$

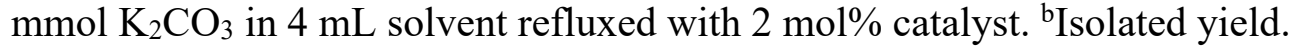

Finally, the catalytic activity of the Ni(II) complexes 3a and $\mathbf{3 b}$ in a Suzuki cross coupling reaction were evaluated using less active bromoarenes and phenyl boronic acid (Scheme 3, Table 2). In the absence of a catalytic amount of $\mathrm{PPh}_{3}$, the yields of the reactions were very low (6-9\%, data not shown). However, in presence of $10 \mathrm{~mol} \% \mathrm{PPh}_{3}$, the yields of the desired product were satisfactory. Such a key role of $\mathrm{PPh}_{3}$ in $\mathrm{Ni}(\mathrm{II})$ catalyzed Suzuki-Miyura cross coupling reaction is not unprecedented. ${ }^{[42]}$

\section{Conclusion}

In summary, we have synthesized 1-( $\underline{n}$-butyl)-3-N-(2furylmethyl/phenylmethyl)acetamido-1, 3-imidazolium chloride which in the presence of base and a $\mathrm{Ni}(\mathrm{II})$ precursor yielded the complexes [1-(n-butyl)-3-N-(furylmethyl/phenyl- 
methyl)acetamido-1,3-imidazol-3-ylidine)] $]_{2} \mathrm{Ni}$. The latter complexes have also been obtained through phosphine derived nickel precursors in which substitution of phosphine takes place by N-heterocyclic carbene. These complexes showed moderate catalytic activities in Suzuki cross coupling reactions.

\section{Acknowledgement:}

One of the authors (RSC) is grateful to DST for the financial support under the DST young scientist scheme YSS/2014/000797. We thank to Mr. Samir Somaiya for encouragement of this work.

\section{Supporting Information}

CCDC 1913414 for 3a and 1913415 for 3b contain the supplementary crystallographic data for this paper. These data can be obtained free of charge from The Cambridge Crystallographic Data Center via www.ccdc.cam.ac.uk/structures. 


\section{Reference:}

1) E. Peris, Chem. Rev. 2018, 118, 9988-10031.

2) L. A. Berben, B. de Bruin, A. F. Heyduk, Chem. Commun. 2015, 51, 1553-1554.

3) B. Zhao, Z. Han, K. Ding, Angew. Chem. Int. Ed. 2013, 52, 4744-4788.

4) V. Blanco, D. A. Leigh, V. Marcos, Chem. Soc. Rev. 2015, 44, 5341-5370.

5) H. V. Huynh, Chem. Soc. Rev. 2018, 118, 9457-9492.

6) F. E Hahn, M. C. Jahnke, Angew. Chem. Int. Ed. 2008, 47, 3122-3172.

7) M. H. Clauberg, D. Schmidt, J. Rust, C. W. Lehmann, N. Arefyeva, M. Wickleder, F. Mohr, J. Organomet. Chem. 2018, 881, 45-50.

8) S. Kumar, A. Narayanan, M. N. Rao, M. M. Shaikh, P. Ghosh, J. Organomet. Chem. 2012, 696, 4159-4165.

9) M. Katari, G. Rajaraman, P. Ghosh, J. Organomet. Chem. 2015, 775, 109-116.

10) S. Ray, M. M. Shaikh, and P. Ghosh, Eur. J. Inorg. Chem. 2009, 1932-1941; M. K. Samantaray, M. M. Shaikh, P. Ghosh, Organometallics 28 (2009) 2267-2275.

11) V. Sriramurthy, O. Kwon, Org. Lett. 2010, 12, 1084-1087.

12) Q. Li, C.H. Ding, X.H. Hou, L.H. Dai, Org. Lett. 2010, 12, 1080-1083.

13) A. P. Prakasham, M. K. Gangwar, P. Ghosh, J. Organomet. Chem. 2018, 859, 106-116.

14) R. Balasubramaniyam, P. Ghosh, Eur. J. Inorg. Chem. 2016, 10, 1448-1465.

15) M. N. Rao, M. Haridas, M. K. Gangwar, P. Rajakannu, A. Ch. Kalita, P. Ghosh, Eur. J. Inorg. Chem. 2015, 9, 1604-1615. 
16) R. Kumar, M. Katari, A. Choudhary, G. Rajaraman, P. Ghosh, Inorg. Chem. 2017, 56, 14859-14869

17) A. Kumar, L. P. Bheeter, M. K. Gangwar, J. B. Sortais, C. Darcel, P. Ghosh, J. Organomet. Chem. 2015, 786, 63-70.

18) D. S. McGuinness, K. J. Cavell, Organometallics 1999, 18, 1596-1605.

19) G. M. Mahandru, G. Liu, J. Montgomery, J. Am. Chem. Soc. 2004, 126, 3698-3699.

20) W. A. Herrmann, G. Gerstberger, and M. Spiegler, Organometallics. 1997, 16, 22092212.

21) V. P. W. Böhm, W.A. Herrmann, Angew. Chem. 2000, 112, 4200; Angew. Chem. Int. Ed. Engl. 2000, 39, 4036.

22) K. Matsubara, K. Ueno and Y. Shibata, Organometallics 2006, 25, 3422-3427.

23) C. Singh, M. K. Gangwar, P. Ghosh, Inorg. Chim. Acta 2017, 466, 358-369.

24) T. Thananatthanachon and M. R. Lecklider, J. Chem. Edu. 2017, 94, 786-789.

25) E. Mas-Marzá, E. Peris, I. Castro-Rodríguez, K. Meyer, Organometallics 2005, 24, 31583162.

26) Crystal Clear-SM Expert v2.1.Rigaku Americas, The Woodlands, Texas, USA, and Rigaku Corporation, Tokyo, Japan, 2015.

27) L. Palatinus, G. Chapuis, J. Appl. Cryst. 2007, 40, 786-790.

28) C. K. Johnson, ORTEP II, Report ORNL-5136, Oak Ridge National Laboratory, Oak Ridge TN, 1976.

29) Y. Koto, F. Shibahara, T. Murai, Org. and Biomol. Chem., 2017, 15, 1810-1820.

30) A. Liske, K. Verlinden, H. Buhl, K. Schaper, C. Ganter, Organometallics 2013, 32, $5269-5272$. 
31) K. Verlinden, H. Buhl, W. Frank and C. Ganter, Eur. J. Inorg. Chem. 2015, 2416-2425.

32) S. Kumar, M. M. Shaikh, P. Ghosh, J. Organomet. Chem. 2009, 694, 4162-4169.

33) C. Preti, G. Tosi, D. De Fillipo, G. Vernani, Can. J. Chem. 1974, 52, 2021-2028.

34) J. N. Liu, B. W. Wu, B. Zhang, Y. C. Liu, Turk. J. Chem. 2006, 30, 41-48.

35) M. A. Ali, A.H. Mirza, J. D. Chartres, P. V Bernhardt, Polyhedron 2011, 30, 299-306.

36) A. J. Carty, Can. J. Chem. 1967, 45, 345-351.

37) R. S. Chauhan, G. Kedarnath, A. Wadawale, A. Munoz-Castro, R. Arratia-Perez, V. K. Jain and W. Kaim, Inorg. Chem., 49 (2010) 4179-4185; R. S. Chauhan, G. Kedarnath, A. Wadawale, A. M. Z. Slawin and V. K. Jain, Dalton Trans., 42 (2013) 259-269; R. S. Chauhan, G. Kedarnath, A. Wadawale, A. L. Rheingold, A. Munoz-Castro, R. ArratiaPerez and V. K. Jain, Organometallics, 31 (2012) 1743-1750; R. S. Chauhan, R. K. Sharma, G. Kedarnath, D. B. Cordes, A. M. Slawin and V.K. Jain, J. Organomet. Chem., 717 (2012) 180-186; R. S. Chauhan, G. Kedarnath, A. Wadawale, D. K. Maity, J. A. Golen, A. L. Rheingold and V. K. Jain, J. Organomet. Chem., 737 (2013) 40-46.

38) M. S. Sanford, M. Ulman, R. H. Grubbs, J. Am. Chem. Soc. 2001, 123, 749-750.

39) T. M. Trnka,R. H. Grubbs, Acc. Chem. Res. 2001, 34, 18-29.

40) A. M. Magill, B. F. Yates, K. J. Cavell, B. W. Skelton, A. H. White, Dalton Trans., 2007, $3398-3406$.

41) N. Kurono, T. Inoue, M. Tokuda Tetrahedron, 2005, 61, 11225-11231

42) T. Tu, H. Mao, C. Herbert, M. Xu and K. Heinz Dötz, Chem. Commun., 2010, 46, $7796-7798$. 
Table 1: $\quad$ Selected crystallographic data for[1-( $\underline{n}$-butyl)-3-N-(2-furylmethyl)acetamido-1,3imidazol-3-ylidine) $]_{2} \mathrm{Ni}$ (3a) and [1-( $\underline{n}$-butyl)-3-N-(2-phenylmethyl)acetamido-1,3-imidazol-3ylidine) $]_{2} \mathrm{Ni}(\mathbf{3 b})$

\begin{tabular}{|c|c|c|}
\hline Complex & 3a & $\mathbf{3 b}$ \\
\hline Chemical formula & $\mathrm{C}_{28} \mathrm{H}_{36} \mathrm{~N}_{6} \mathrm{NiO}_{4}$ & $\mathrm{C}_{32} \mathrm{H}_{40} \mathrm{~N}_{6} \mathrm{NiO}_{2}$ \\
\hline Formula wt. & 579.33 & 599.41 \\
\hline Crystal size $\left(\mathrm{mm}^{3}\right)$ & $0.15 \times 0.10 \times 0.08$ & $0.070 \times 0.030 \times 0.020$ \\
\hline Crystal system & Orthorhombic & Monoclinic \\
\hline Space group & Pccn & $P 21 / n$ \\
\hline $\mathrm{a}(\AA)$ & $10.1795(2)$ & $9.8093(4)$ \\
\hline $\mathrm{b}(\AA)$ & $16.4812(3)$ & $16.8345(6)$ \\
\hline $\mathrm{c}(\AA)$ & $16.6731(4)$ & $17.7177(5)$ \\
\hline$\beta\left(^{\circ}\right)$ & 90.0 & $95.204(3)$ \\
\hline Volume $\left(\AA^{3}\right)$ & $2797.25(10)$ & $2913.75(18)$ \\
\hline$\rho_{\text {cacld }}, \mathrm{g} \mathrm{cm}^{-3}$ & 1.376 & 1.366 \\
\hline Z & 4 & 4 \\
\hline$\mu\left(\mathrm{mm}^{-1}\right) / \mathrm{F}(000)$ & $0.738 / 1224$ & $0.707 / 1272$ \\
\hline No of reflections collected & 22516 & 37529 \\
\hline No of independent reflection $\left(R_{\text {int }}\right)$ & $3210(0.0245)$ & $6757(0.0975)$ \\
\hline Data/restraints/parameters & $3210 / 0 / 178$ & $6757 / 38 / 391$ \\
\hline Final $\mathrm{R}_{1}$, wR $\mathrm{R}_{2}$ indices $(I>2 \sigma I)$ & $0.0275 / 0.0709$ & $0.0480 / 0.0825$ \\
\hline
\end{tabular}




\begin{tabular}{l|c|c}
\hline $\mathrm{R}_{1}, \mathrm{wR}_{2}$ (all data) & $0.0347 / 0.0736$ & $0.1280 / 0.0990$ \\
Goodness of fit on $\mathrm{F}^{2}$ & 1.054 & 0.983 \\
\hline
\end{tabular}

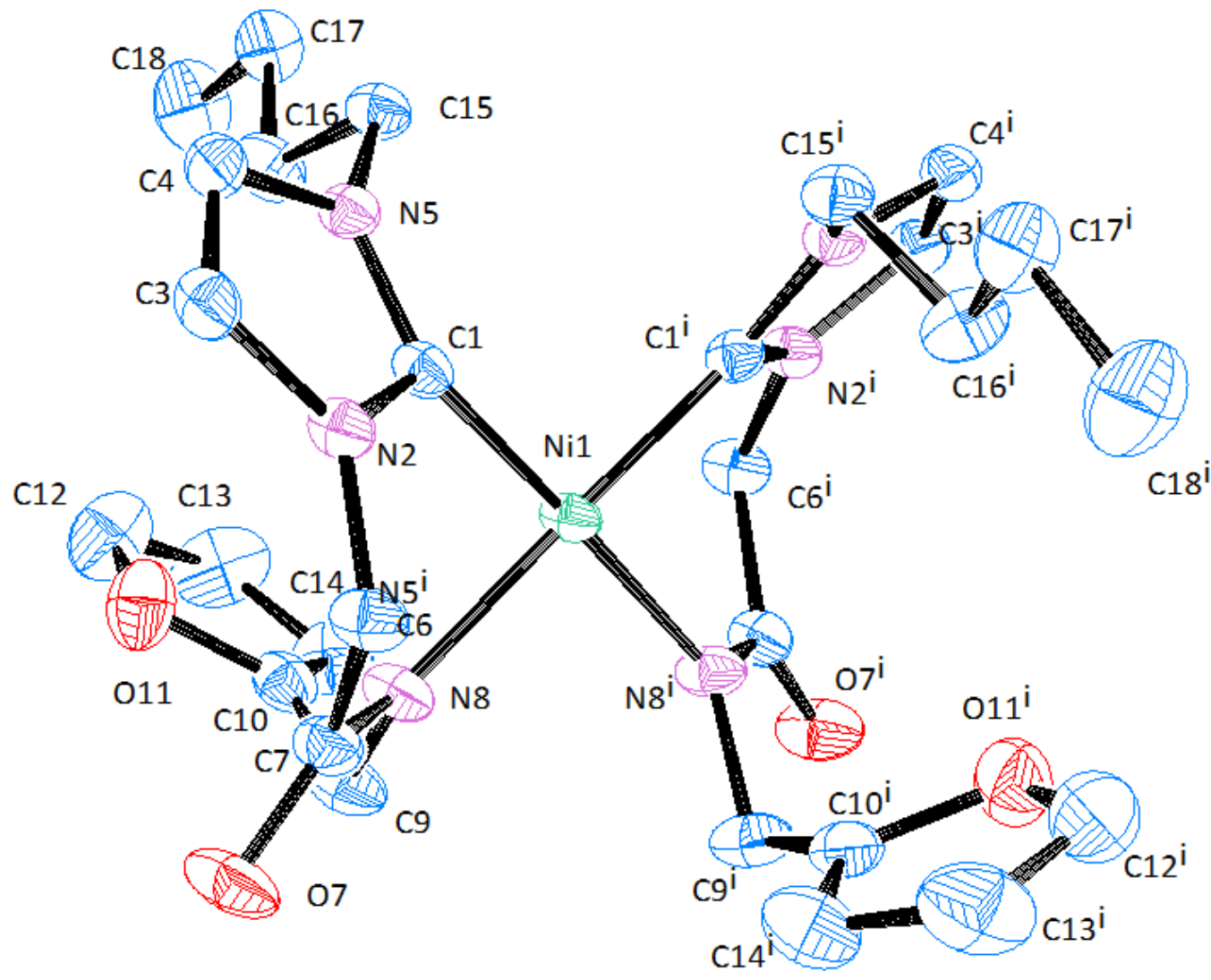

Figure 2: ORTEP diagram of [1-( $\underline{n}$-butyl)-3-N-(2-furylmethyl)acetamido-1,3-imidazol-3ylidine) $]_{2} \mathrm{Ni}$ (3a) with $50 \%$ probability level. Selected bond lengths $(\AA)$ and bond angles $\left(^{\circ}\right)$ : Ni(1)-C(1) 1.8658(13), Ni(1)-N(8)- 1.9297(11); C(1)-Ni(1)-C(1) 96.05(8), C(1)-Ni (1)-N(8) 176.55(5), C(1)-Ni(1)-N(8) 86.58(5), N(8)-Ni(1)-N(8) 90.87(7). 


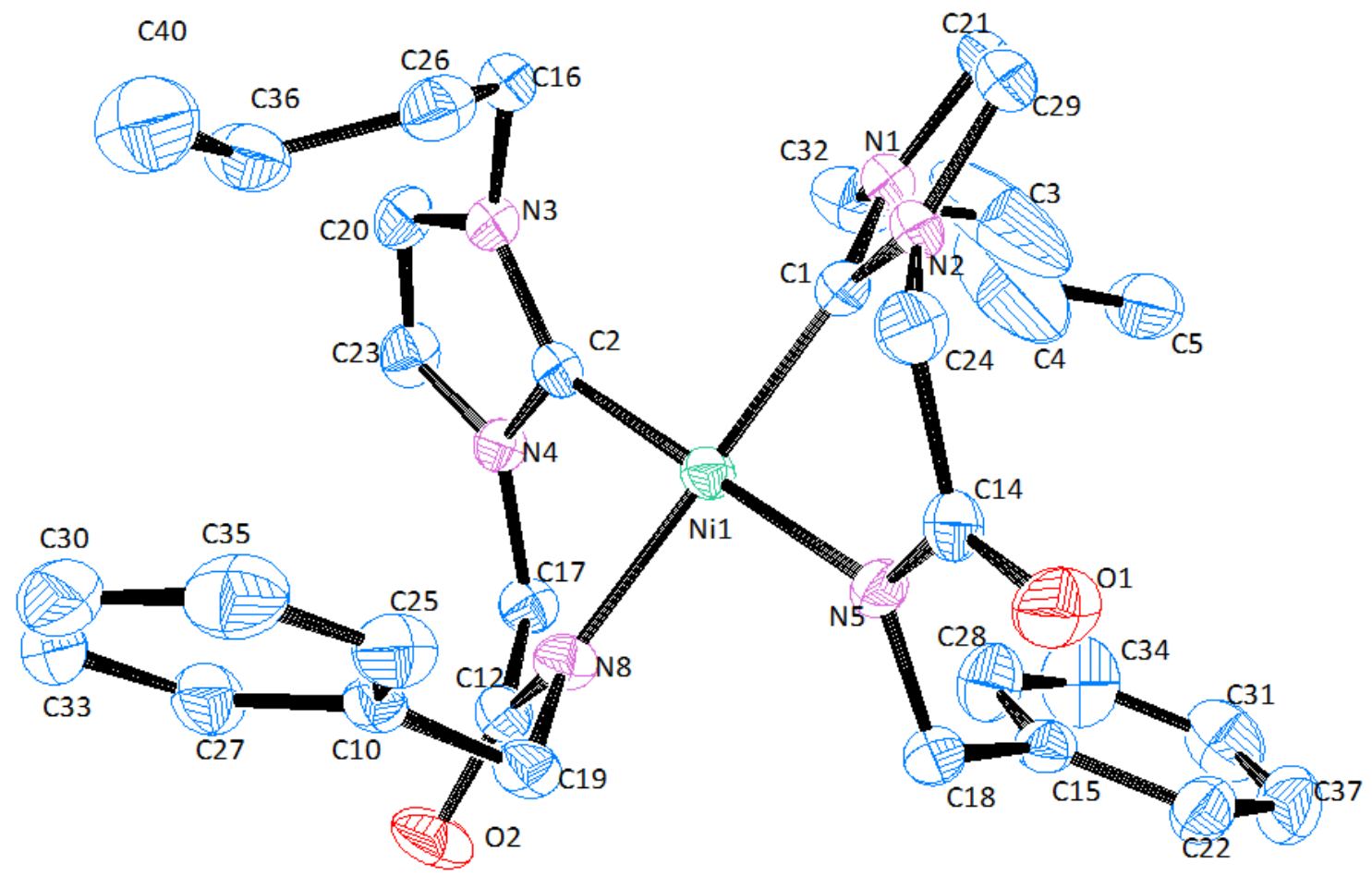

Figure 3: $\quad$ ORTEP diagram of [1-( $\underline{n}$-butyl)-3-N-(2-phenylmethyl)acetamido-1,3-imidazol-3ylidine) $]_{2} \mathrm{Ni}(3 \mathrm{~b})$ with $50 \%$ probability level. Selected bond lengths $(\AA)$ and bond angles $\left(^{\circ}\right)$ : Ni(1)-C(1) 1.861(2), Ni(1)-C(21) 1.859(2), Ni(1)-N(8) 1.9306(19), Ni(1)-N(28) 1.9367(19); C(1)-Ni(1)-C(21) 92.33(10), N(8)-Ni(1)-N(28) 93.13(8), C(1)-Ni(1)-N(8) 87.35(9), C(21)-Ni(1)$\mathrm{N}(28)$ 87.21(10), C(21)-Ni(1)-N(8) 178.90(9), C(1)-Ni(1)-N(28) 179.32(19). 
